Pacific Journal of Mathematics

THE CHARACTERISTIC POLYNOMIAL OF THE 


\title{
THE CHARACTERISTIC POLYNOMIAL OF THE MONODROMY
}

\author{
Alan H. Durfee
}

\begin{abstract}
This paper contains miscellaneous results about the monodromy of a singularity of an algebraic curve $f\left(z_{0}, z_{1}\right)$, particularly its characteristic polynomial, and its relation to branched cyclic covers of the link of the singularity.
\end{abstract}

Let $f\left(z_{0}, \cdots, z_{n}\right)$ for $n \geqq 0$ be a complex polynomial vanishing at the origin, and suppose that $f$ has at most an isolated critical point there. The link of this singularity is the manifold $K=f^{-1}(0) \cap$ $S^{2 n+1} \subset S^{2 n+1}$, where $S^{2 n+1}$ is a sufficiently small sphere centered at the origin. $K$ is an $(n-2)$-connected $(2 n-1)$-manifold. For $\delta \neq 0$, $f^{-1}(\delta)$ intersected with the corresponding small ball is a smooth $2 n$ manifold $F$ whose boundary is diffeomorphic to $K$. Letting $\delta$ travel once about the origin in the positive direction induces a monodromy automorphism $h: \widetilde{H}_{n}(F) \rightarrow \widetilde{H}_{n}(F)$. The homology group $\widetilde{H}_{n-1}(K)$ of the link is isomorphic to the cokernel of $I-h$ [11, Theorem 8.5]. We will be particularly interested in the characteristic polynomial $\Delta(t)=\operatorname{det}(I t-h)$ of the monodromy. For these topics, see [11].

For fixed $f$, we let $K_{k} \subset S^{2 n+3}$ be the link of the polynomial $f\left(z_{0}, \cdots, z_{n}\right)+z_{n+1}^{k} . \quad K_{k}$ is thus an $(n-1)$-connected $(2 n+1)$-manifold. It is well-known that $K_{k}$ is the $k$-fold cyclic cover of $S^{2 n+1}$ branched along $K[6]$.

In the first section we relate the rank and 2-torsion of $H_{1}\left(K_{2}\right)$ to the number of branches of $f\left(z_{0}, z_{1}\right)$ at the singular point. As an example, we compute $H_{1}\left(K_{2}\right)$ of the singularity $\left(z_{0}+z_{1}^{2}\right)\left(z_{0}^{2}+z_{1}^{5}\right)$ used to foliate odd dimensional spheres [4], thus avoiding resolution of singularities. Section two reproves an old result of Zariski [18] on computing the rank of $H_{1}\left(K_{k}\right)$ in terms of $\Delta(t)$ (which is closely related to the Alexander polynomial of the link). The rest of the section is a digression on the roots \pm 1 of $\Delta(t)$. Section three combines the results of the previous sections to give a simple criterion for the monodromy of $f\left(z_{0}, z_{1}\right)$ to be of infinite order, namely, that -1 be a root of $\Delta(t)$ of multiplicity greater than or equal to the number of branches of $f$ at the singular point. In particular, $\left(z_{0}+z_{1}^{2}\right)\left(z_{1}^{2}+z_{2}^{5}\right)$ satisfies this criterion. The first example of monodromy of infinite order was found by A'Campo [1]. Shortly thereafter the author found a mistake in a preliminary version of [4]; this mistake generalized to the present paper. The results here have been expanded by Woods [19]. For an analytic approach to 
monodromy of infinite order, see [5].

1. The two-fold branched cyclic cover. Let $f\left(z_{0}, \cdots, z_{n}\right)$ be a complex polynomial as above, with link $K \subset S^{2 n+1}$. Let $K_{2}$ be the 2-fold cyclic cover of $S^{2 n+1}$ branched along $K$. We claim that the rank plus the number of 2-torsion coefficients of $\widetilde{H}_{n-1}(K)$ is equal to the rank plus the number of 2-torsion coefficients of $H_{n}\left(K_{2}\right)$ : First consider the polynomial $f^{\prime}\left(z_{n+1}\right)=z_{n+1}^{2}$ in one variable. The monodromy $h^{\prime}$ of $f^{\prime}$ on $\widetilde{H}_{0}(F) \simeq Z$ may be calculated directly, and turns out to be multiplication by -1 . (See for example [11, §9].) Let $h^{\prime \prime}$ be the monodromy of the polynomial $f+f^{\prime}$. By [16], $h^{\prime \prime}$ is equivalent to $h \otimes h^{\prime}=-h$. Since $\tilde{H}_{n-1}(K) \simeq \operatorname{cok}(I-h)$ and $H_{n}\left(K_{k}\right) \simeq \operatorname{cok}\left(I-h^{\prime \prime}\right)$, we have $\widetilde{H}_{n-1}(K) \otimes Z_{2} \simeq H_{n}\left(K_{2}\right) \otimes Z_{2}$, which proves the italicized assertion.

Next specialize to the case $n=1$. Let $r$ be the number of branches of $f\left(z_{0}, z_{1}\right)$ at the origin, so that $K \subset S^{3}$ has $r$ components. We have proved the following result.

Proposition 1. When $n=1$, the rank plus the number of 2torsion coefficients of $H_{1}\left(K_{2}\right)$ is $r-1$.

The italicized assertion above is true for any simple knot $K \subset S^{2 n+1}$ and $K_{2} \subset S^{2 n+3}$ its suspension, a knot with the same Seifert matrix $\mathscr{L}$ up to sign as $K$ [2]. The homology groups of $K$ and $K_{2}$ are determined by $\mathscr{L} \pm \mathscr{L}^{t}$, and the same proof works. When $n=1$, the proposition generalizes to $H_{1}\left(K_{k}\right) \leqq(k-1)(r-1)$, for arbitrary links $K \subset S^{3}$ of $r$ components, and $K_{k}$ the branched $k$-fold cyclic cover, for $k$ prime [17, Corollary 5].

For example, consider the singularity $f\left(z_{0}, z_{1}\right)=\left(z_{0}+z_{1}^{2}\right)\left(z_{0}^{2}+z_{1}^{5}\right)$. To foliate odd-dimensional spheres as in [4], the key point is that $H_{1}\left(K_{2}\right) \simeq Z$; here we find this group without resolving the singularity $f\left(z_{0}, z_{1}\right)+z_{2}^{2}$. The polynomial $f$ has two branches at the origin, and its link $K \subset S^{3}$ is a torus knot of type $(2,5)$ together with an unknotted circle twice linking its core (see for example [14, §2.3]):

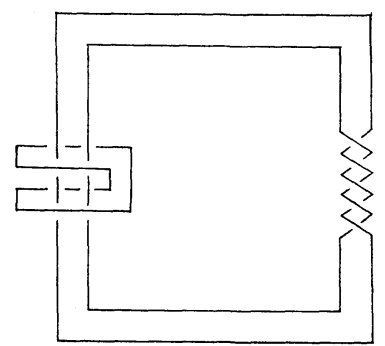

As remarked above, $K_{2}$ is the 2 -fold cyclic cover of $S^{3}$ branched along this link. The torsion subgroup of $H_{1}(K)$ is isomorphic to the 
cokernel of its quadratic form [8]. This quadratic form is computed by coloring alternate regions black and white; by row and column operations its cokernel is seen to have no torsion. In particular, $H_{1}\left(K_{2}\right)$ has no 2 -torsion, so by the proposition, its rank is one. Thus $H_{1}\left(K_{2}\right) \simeq \boldsymbol{Z}$.

Do all the possibilities of the proposition occur? If $f\left(z_{0}, z_{1}\right)=$ $z_{0}^{2}+z_{1}^{2}, H_{1}\left(K_{2}\right) \simeq Z_{2}$. Thus if $r=2$, both possibilities occur. If $r=3$ there are three possibilities, all of which do occur: If $f\left(z_{0}, z_{1}\right)=$ $z_{0}^{3}+z_{1}^{3}$, then $H_{1}\left(K_{2}\right) \simeq Z_{2} \oplus Z_{2}$, if $f\left(z_{0}, z_{1}\right)=\left(z_{0}+z_{1}^{2}\right)\left(z_{0}-z_{1}^{2}\right)\left(z_{0}^{2}+z_{1}^{5}\right)$, then $H_{1}\left(K_{2}\right) \simeq \boldsymbol{Z} \oplus \boldsymbol{Z}_{4}$, and if $f\left(z_{0}, z_{1}\right)=z_{0}^{6}+z_{1}^{3}$, then $H_{1}\left(K_{2}\right) \simeq \boldsymbol{Z} \oplus \boldsymbol{Z}$. (Also see $[5, \S 1]$.)

2. The characteristic polynomial of the monodromy. Again fix a complex polynomial $f\left(z_{0}, \cdots, z_{n}\right)$ as above, with link $K \subset S^{2 n+1}$, monodromy $h$, and characteristic polynomial $\Delta(t)=\operatorname{det}(I t-h)$. Orlik $[13$, p. 264-5] has noticed that if the monodromy is of finite order, then the rank of $\widetilde{H}_{n-1}(K)$ is the multiplicity of 1 as a root of $\Delta(t)$.

Proof. Let $\Delta_{1}(t), \cdots, \Delta_{n}(t)$ be the elementary divisors of $I t-h$ over $Q[t]$. These have the usual properties: (1) $\Delta_{1}(t)\left|\Delta_{2}(t)\right| \cdots \mid \Delta_{r}(t)$; (2) $\Delta_{r}(t)$ is the minimum polynomial of $h$; (3) $\Delta(t)=\Delta_{1}(t) \Delta_{2}(t) \cdots \Delta_{r}(t)$; and (4) $\operatorname{cok}(I t-h) \cong \operatorname{cok} D(t)$ as $Q[t]$-modules, where $D(t)$ has a diagonal matrix with entries $1, \cdots, 1, \Delta_{1}(t), \cdots, \Delta_{r}(t)$. Since $h$ is of finite order, there is an $N$ such that $h^{N}=I$. Thus by $(2), \Delta_{r}(t) \mid\left(t^{N}-1\right)$. Hence the factorization in $\boldsymbol{Q}[t]$ of $\Delta_{r}[t]$ has no repeated factors; by $(1)$, this is true for $\Delta_{1}(t), \cdots, \Delta_{r-1}(t)$ as well. In particular, the factor $(t-1)$ can occur at most once. Recall that $\widetilde{H}_{n-1}(K) \cong \operatorname{cok}(I-h)$. Setting $t=1$ in (4), we obtain the $\boldsymbol{Q}$-module isomorphism $\widetilde{H}_{n-1}(K) \cong$ cok $D(1)$. By (3) and the above, the rank of cok $D(1)$ is equal to the multiplicity of $(t-1)$ in the factorization of $\Delta(t)$.)

We apply this observation to the $k$-fold branched cyclic cover $K_{k}$.

Proposition 2. Suppose that the monodromy of $f\left(z_{0}, \cdots, z_{n}\right)$ is of finite order. Then the rank of $\tilde{H}_{n}\left(K_{k}\right)$ is equal to the number (counted with multiplicities) of roots $\alpha \neq 1$ of $\Delta(t)$ with $\alpha^{k}=1$.

When $n=1$ and $f\left(z_{0}, z_{1}\right)$ has one branch at the origin, $\Delta(t)$ is then the Alexander polynomial of the knot $K \subset S^{3}$ [11, Lemma 10.1], and Zariski [18] showed that $H_{1}\left(K_{k}\right)$ could be computed in this fashion. Since Lê [9] has shown that such $f$ have monodromy of finite order, our result implies Zariski's. Also see [17].

Proof. First consider the polynomial $f^{\prime}\left(z_{n+1}\right)=z_{n+1}^{k}$ in one variable. The monodromy $h^{\prime}$ of $f^{\prime}$ may be calculated directly, and its charac- 
teristic polynomial $\Delta^{\prime}(t)$ turns out to be $t^{k-1}+\cdots+t+1=\prod_{j=1}^{k-1}\left(t-\eta^{j}\right)$, where $\eta$ is a primitive $k^{\text {th }}$ root of unity. (See for example [11, §9].)

Let $h^{\prime \prime}$ be the monodromy associated to the polynomial $f+f^{\prime}$, and let $\Delta^{\prime \prime}(t)$ be its characteristic polynomial. By [16], $h^{\prime \prime}$ is equivalent to $h \otimes h^{\prime}$. Suppose $\Delta(t)$ factors over the complex numbers as $\Pi_{i}\left(t-\alpha_{i}\right)$. Then $\Delta^{\prime \prime}(t)=\Pi_{i, j}\left(t-\alpha_{i} \eta^{j}\right)$. Hence 1 is a root of $\Delta^{\prime \prime}(t)$ precisely when there is a $j, 1 \leqq j \leqq k-1$, and an $\alpha_{i}$ such that $\alpha_{i} \eta^{j}=1$; this will occur if and only if $\alpha_{i}$ is a $k^{\text {th }}$ root of unity different from 1. This proves the proposition.

The rest of this section is a diversion about the roots \pm 1 of $\Delta(t)$ when $n=1$. Let $r$ be the number of branches of $f\left(z_{0}, z_{1}\right)$ at the origin (the number of components of its link $K \subset S^{3}$ ).

Proposition 3. (i) The multiplicity of 1 as a root of $\Delta(t)$ is $r-1$.

(ii) The multiplicity of -1 as a root of $\Delta(t)$ is even.

Part (i) says that Orlik's assertion at the beginning of this section is true when $n=1$, even if the monodromy is of infinite order. When $n=2$, the singularity $\left(z_{0}+z_{1}^{2}\right)\left(z_{0}^{2}+z_{1}^{5}\right)+z_{2}^{2}$ is a counterexample to (i) (with $r-1$ replaced by rank $H_{1}(K)$ ) and (ii). (See $\S \S 1$ and 3.) Both parts are true for arbitrary knots $K \subset S^{3}$ when $r=1$.

Proof. If $r=1, \Delta(t)$ is (up to a factor of $\pm t^{i}$ ) the Alexander polynomial of the link of $f\left(z_{0}, z_{1}\right)$ [11, Lemma 10.1], and it is well known that the value of this polynomial at 1 is \pm 1 . Hence the proposition is true if $r=1$. If $r \geqq 2$, then again according to the above reference, $\pm t^{i} \Delta(t)=(t-1) \Delta(t, \cdots, t)$, where $\Delta\left(t_{1}, \cdots, t_{r}\right)$ is the Alexander polynomial of the link. Let $\nabla(t)=(t-1)^{2-r} \Delta(t, \cdots, t)$ be the polynomial defined by Hosokawa [7]. It is shown that $\pm V(1)$ is the $(r-1)$-minor determinant of an $r \times r$ matrix of rank $(r-1)$ whose $i j^{\text {th }}$ entry is the linking number $l_{i j}$ of the $i^{\text {th }}$ and $j^{\text {th }}$ components of the link, $i \neq j$, and whose $i^{\text {th }}$ diagonal entry is $-\sum_{1 \leqq j \leqq r, i \neq j} l_{i j}$. We claim that $\nabla(1) \neq 0$. In fact, $l_{i j}, i \neq j$, is also the intersection number of the $i^{\text {th }}$ and $j^{\text {th }}$ branch, which is positive [14]. Thus the upper left-hand $(r-1)$-minor satisfies conditions (a), (b), (c) and (d) of $[12, p .6]$ and hence is negative definite as a quadratic form. Hence its determinant, which is $\pm \nabla(1)$, is nonzero. Since $\Delta(t)=$ $\pm t^{i}(t-1)^{r-1} V(t), 1$ is a root of $\Delta(t)$ of multiplicity $r-1$. This proves part (i).

When $r=1, \nabla(t)$ is defined to be the Alexander polynomial. Hence for all $r \geqq 1, \Delta(t)= \pm t^{i}(t-1)^{r-1} \nabla(t)$, so it suffices to show that -1 is a root of $\nabla(t)$ of even multiplicity. In [7] it is proved 
that $\nabla(t)$ is symmetric, that is, $\nabla(t)=t^{d} \nabla\left(t^{-1}\right)$ where $d$ is the degree of $\nabla(t)$, and that $d$ is even. Now 0 is not a root of $\nabla(t)$. The symmetry implies that if $c$ is a root, then so is $c^{-1}$. Thus in the factorization of $\nabla(t)$ over the complex numbers, the factor $(t-c)$ may be paired with the factor $\left(t-c^{-1}\right)$, except possibly when $c= \pm 1$. Since $c \neq 1$ by part (i), and there are an even number of factors, the factor $(t+1)$ must occur an even number of times. This proves part (ii).

3. Monodromy of infinite order. Let $f\left(z_{0}, z_{1}\right)$ be a polynomial as above with $r$ branches at the origin (so that its link $K \subset S^{3}$ has $r$ components), and let $\Delta(t)$ be the characteristic polynomial of its monodromy.

Proposition 4. The monodromy of $f\left(z_{0}, z_{1}\right)$ is of infinite order if -1 is a root of $\Delta(t)$ of multiplicity $\geqq r$.

Proof. If the monodromy were of finite order, then by Proposition 2 this multiplicity would be the rank of $H_{1}\left(K_{2}\right)$, which by Proposition 1 is $\leqq r-1$.

The Alexander polynomials of all compound torus links with two components have been computed by Burau [3]. (Burau actually computes the Reidemeister-Schumann polynomial, the determinant of a presentation matrix of $H_{1}(\widetilde{X})$, where $\tilde{X}$ is the universal abelian cover of $S^{3}-K$. Levine $[10, \S 8]$ shows that this is the same as the usual Alexander polynomial. Alternatively, R.H. Fox (private correspondence) applies the free differential calculus to Reidemeister and Schumann's presentation of $\pi_{1}\left(S^{3}-K\right)$ and shows that the $(d+1)^{\text {st }}$ elementary ideal of the resulting matrix is the same as the $d^{\text {th }}$ elementary ideal of the presentation matrix (7) in $\left[15\right.$, p. 258] of $H_{1}(\widetilde{X})$ derived from this. Hence the Alexander polynomial is the same as the determinant of this matrix. He continues, "The calculations in the two Burau papers are almost too painful to contemplate, but I am sure that the results are correct...".)

For example, $f\left(z_{0}, z_{1}\right)=\left(z_{0}+z_{1}^{2}\right)\left(z_{0}^{2}+z_{1}^{5}\right)$ has monodromy of infinite order: The curve $f=0$ has two branches at the origin, and according to Burau its Alexander polynomial is

$$
\Delta(x, y)=\frac{\left(1-x^{2} y^{4}\right)\left(1-x^{4} y^{10}\right)}{\left(1-x y^{2}\right)\left(1-x^{2} y^{5}\right)} .
$$

The characteristic polynomial of the monodromy is then $\Delta(t)=$ $\pm(t-1)\left(1+t^{3}\right)\left(1+t^{7}\right)$ by [11, Lemma 10.1], so -1 is a root of $\Delta(t)$ of multiplicity 2 . 


\section{REFERENCES}

1. N. A'Campo, Sur la monodromie des singularités isolées d'hypersurfaces complexes, Inventions Math., 20 (1973), 147-169.

2. G. Bredon, Regular $0(n)$-manifolds, suspension of knots, and knot periodicity, Bull. Amer. Math. Soc,, 79 (1973), 87-91.

3. W. Burau, Kennzeichnung der Schlauchverkettungen, Abh. Math. Sem. Hamburg, 10 (1934), 285-297. ${ }^{1}$

4. A. Durfee, Foliations of odd-dimensional spheres, Annals of Math., 96 (1972), 407411.

5. - The mondromy of a degenerating family of curves, appeared, in Inventiones Math.

6. A. Durfee and L. Kauffman, Periodicity of branched cyclic covers, to appear, Math. Annalen.

7. F. Hosokawa, On V-polynomials of links, Osaka Math. J., 10 (1958), 273-282.

8. R. Kyle, Branched covering spaces and the quadratic form of links, Annals of Math., 59 (1954), 539-548.

9. Lê Dũng Tráng, Sur les noeuds algébrique, Centre de Math. Ecole Polytechnique, Paris, 1971.

10. J. Levine, A method for generating link polynomials, Amer. J. Math., 89 (1967), 69-84.

11. J. Milnor, Singular points of complex hypersurfaces, Annals of Math Studies, no. 61, Princeton University Press, 1968.

12. D. Mumford, The topology of normal singularities of an algebraic surface and a criterion for simplicity, Publ. Math., no. 9, l'Inst. des Hautes Études Sci., Paris, 1961. 13. P. Orlik, On the homology of weighted homogeneous manifolds, Proceedings of the second conference on compact transformation groups, Lecture notes in math. vol. 298, p. 260-269, Springer (1972).

14. J. Reeve, A summary of results in the topological classification of plane algebroid singularities, Rendiconti Sem. Mat. Torio, 14 (1954-55), 159-187.

15. K. Reidemeister and H. Schumann, L-Polynome von Verkettungen, Abh. Math. Sem. Hamburg, 10 (134), 256-262.

16. M. Sebastiani and $\mathrm{R}$. Thom, Un résultat sur la monodromie, Inventiones Math., 13 (1971), 90-96.

17. D. Sumners, On the homology of finite cyclic coverings of higher dimensional links, Proc. Am. Math. Soc., 45 (1974), no. 3.

18. O. Zariski, On the topology of algebroid singularities, Amer. J. Math., 54 (1932), $453-465$.

19. J. Woods, Some criteria for finite and infinite monodromy of plane algebraic curves, Inventiones Math., 26 (1974), 179-185.

Received December 2, 1974. Research partially supported by National Science Foundation Grants GP $34785 \mathrm{X}$ and MPS 72-05055 A02.

UNIVERSITY OF WASHINGTON

AND

Institute FOR ADVANCed Study

${ }^{1}$ Beware of the following misprints: $(n, m)$ on p. 295 , line 6 should be $\left(n^{\prime}, m^{\prime}\right)$, and $n$ in line 10 should be $n^{\prime}$. 


\section{PACIFIC JOURNAL OF MATHEMATICS}

\section{EDITORS}

RICHARD ARENS (Managing Editor) University of California

Los Angeles, California 90024

\section{R. A. Beaumont}

University of Washington Seattle, Washington 98105
J. DugundJI Department of Mathematics University of Southern Californıa Los Angeles, California 90007

D. Gilbarg aNd J. Milgram Stanford University Stanford, California 94305

\section{ASSOCIATE EDITORS}

E. F. BeCKENBACH

B. H. NeUmanN

F. Wolf

K. YoSHIDA

\section{SUPPORTING INSTITUTIONS}

UNIVERSITY OF BRITISH COLUMBIA CALIFORNIA INSTITUTE OF TECHNOLOGY UNIVERSITY OF CALIFORNIA MONTANA STATE UNIVERSITY UNIVERSITY OF NEVADA NEW MEXICO STATE UNIVERSITY OREGON STATE UNIVERSITY UNIVERSITY OF OREGON OSAKA UNIVERSITY
UNIVERSITY OF SOUTHERN CALIFORNIA STANFORD UNIVERSITY UNIVERSITY OF TOKYO UNIVERSITY OF UTAH WASHINGTON STATE UNIVERSITY UNIVERSITY OF WASHINGTON AMERICAN MATHEMATICAL SOCIETY NAVAL WEAPONS CENTER 


\section{Pacific Journal of Mathematics \\ Vol. 59, No. 1 \\ May, 1975}

Shashi Prabha Arya and M. K. Singal, More sum theorems for topological

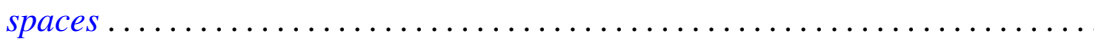

Goro Azumaya, F. Mbuntum and Kalathoor Varadarajan, On M-projective and

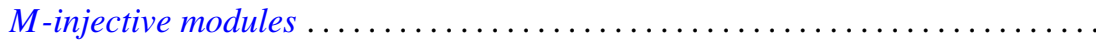

Kong Ming Chong, Spectral inequalities involving the infima and suprema of

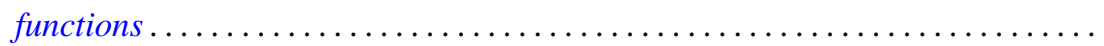

Alan Hetherington Durfee, The characteristic polynomial of the monodromy .......

Emilio Gagliardo and Clifford Alfons Kottman, Fixed points for orientation

preserving homeomorphisms of the plane which interchange two points ......

Raymond F. Gittings, Finite-to-one open maps of generalized metric spaces .......

Andrew M. W. Glass, W. Charles (Wilbur) Holland Jr. and Stephen H. McCleary,

$a^{*}$-closures of completely distributive lattice-ordered groups .............

Matthew Gould, Endomorphism and automorphism structure of direct squares of

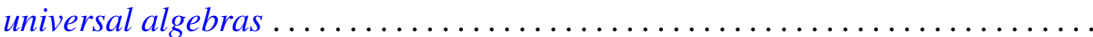

R. E. Harrell and Les Andrew Karlovitz, On tree structures in Banach spaces .....

Julien O. Hennefeld, Finding a maximal subalgebra on which the two Arens

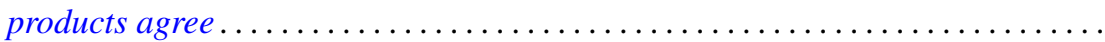

William Francis Keigher, Adjunctions and comonads in differential algebra .......

Robert Bernard Kelman, A Dirichlet-Jordan theorem for dual trigonometric

series

Allan Morton Krall, Stieltjes differential-boundary operators. III. Multivalued operators-linear relations...

Hui-Hsiung Kuo, On Gross differentiation on Banach spaces .

Tom Louton, A theorem on simultaneous observability ...

Kenneth Mandelberg, Amitsur cohomology for certain extensions of rings of algebraic integers.

Coy Lewis May, Automorphisms of compact Klein surfaces with boundary . . .

Peter A. McCoy, Generalized axisymmetric elliptic functions .

211

Muril Lynn Robertson, Concerning Siu's method for solving $y^{\prime}(t)=F(t$, $y(g(t)))$. .

Richard Lewis Roth, On restricting irreducible characters to normal

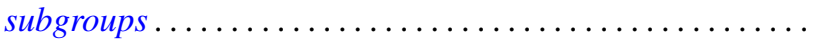

Albert Oscar Shar, $P$-primary decomposition of maps into an $H$-space .

Kenneth Barry Stolarsky, The sum of the distances to certain pointsets on the unit circle.

Bert Alan Taylor, Components of zero sets of analytic functions in $C^{2}$ in the unit ball or polydisc

Michel Valadier, Convex integrands on Souslin locally convex spaces ...

Januario Varela, Fields of automorphisms and derivations of $C$

Arnold Lewis Villone, A class of symmetric differential operators with deficiency

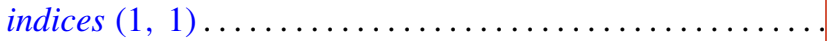

